\section{A third way for lysosomal treatments}

\author{
By Tim Fulmer, Staff Writer
}

Scientists at The Scripps Research Institute have found that generic small-molecule calcium channel blockers for hypertension can restore enzyme function in lysosomal storage disorders. But human testing is needed to determine how they fit in with the expensive therapies used to treat these disorders.

In their research, reported in PLoS Biology, the Scripps team shows that diltiazem and verapamil, two generic small-molecule calcium channel blockers, partially restore enzyme function in three different lysosomal storage disorder (LSD) cell lines by altering cellular calcium levels. ${ }^{1}$

LSDs include more than 40 genetic disorders that result from mutations in and reduced activity of a lysosomal enzyme, and the gradual accumulation of its substrate in the lysosome. Often the mutations cause misfolding of the enzyme in the endoplasmic reticulum (ER), with subsequent degradation by the proteasome.

In Gaucher's disease, for example, the culprit is misfolded glucocerebrosidase. Two key mutations that cause the misfolding are N370S, which is associated with type 1 Gaucher's disease, and L444P, which is linked to type 2 and type 3 Gaucher's. The latter two forms of the disease affect the CNS.
"Very little would be gained by performing further preclinical studies, and I believe the next step would be to perform clinical trials in patients with neuropathic disease and in patients homozygous for the N370S mutation who are not receiving enzyme replacement therapy." -Ernest Beutler,
The Scripps Research Institute

toms-type 3 Gaucher's disease and late-onset Tay-Sachs disease-“did not generate significant results."

nction of the endogenous enzyme.

Previous work has suggested a link between altered intracellular calor of chemistry at Scripps and principal investigato restore mutant enzyme function in LSDs.

One unanswered question is the mechanism by which calcium channel restore the folding and activity of mutant enzymes in LSDs."

Kelly added that a single calcium channel blocker could potentially e used to treat a spectrum of LSDs. "Because compounds like diltiazem improve mutant enzyme function not through direct binding to the enzyme but through the more general mechanism of altering cellular calcium homeostasis, they have potential to treat a variety of LSDs," he said. "Given diltiazem's known ability to cross the blood-brain barrier, the compound seems to be suited to treat neuropathic LSDs" such as type 2 or type 3 Gaucher's and mucopolysaccharidosis IIIA (MPS IIIA).

In fibroblasts derived from patients with nonneuropathic and neuropathic forms of Gaucher's disease, diltiazem partially restored glucocerebrosidase activity by 1.5-3.5-fold compared with untreated controls. Both diltiazem and verapamil also improved mutant enzyme function in fibroblasts from patients with two other LSDs: $\alpha$-mannosidosis and MPS IIIA.

The improvements in enzymatic activity correlated with the blockage of plasma membrane calcium ion channels and the lowering of calcium influx.

Although fibroblast models are often used in in vitro studies of LSDs, Emil Kakkis, SVP and CMO at BioMarin Pharmaceutical Inc., noted, "It is not clear whether a twofold increase in activity over a deficient level is sufficient to be therapeutic."

Thus, he said, "further in vitro characterization of the calcium channel blockers ought to include cell types other than fibroblasts, particularly cells that have high glucocerebroside content in disease, such as macrophages. This would presumably show whether the twofold activity increase that Kelly sees in fibroblasts translates to cell types where baseline levels of storage are quite high and perhaps only reversible through enzyme replacement therapy."

BioMarin markets the enzyme replacement therapies Aldurazyme laronidase and Aryplase galsulfase to treat MPS I (Hurler's syndrome) and MPS IV, respectively. 


\section{TARGETS \& MECHANISMS}

Tim Edmunds, VP of therapeutic protein research at Genzyme, said the company has seen "a similar twofold restoration of activity of the N370S glucocerebrosidase mutant in rodent macrophages treated with the chemical chaperones $N$-nonyl-deoxynojirimycin (NN-DNJ) and isofagomine. However, the mechanism of restoration was clearly different from Kelly's interpretation. Our compounds acted by preventing degradation of mutant enzyme in the lysosome, not by promoting folding via ER pathways."

$\mathrm{NN}-\mathrm{DNJ}$ and isofagomine are generic iminosugar compounds.

Edmunds pointed out that multiple types of enzymatic mutations give rise to storage disorders, including trafficking mutations, folding mutations and catalytic mutations. He suggested that each of these types of mutations will likely require different strategies for restoring function. For example, Edmunds said, in some cases restoration may involve preventing degradation of mutant enzyme in the lysosome, whereas in other instances, rescue may occur through folding pathways in the ER.

"Identifying the appropriate small-molecule chaperone to restore a given type of mutation could be quite costly, time consuming and have regulatory risk," said Edmunds. Thus, regarding small molecules, he said Genzyme's preferred approach "has been to develop small-molecule substrate reduction therapies which presumably have a more universal mechanism than chaperone therapies."

Genzyme's Genz-112638, a ceramide analog that inhibits glucosylceramide synthase, is a substrate reduction therapy that is in a Phase II trial to treat type 1 Gaucher's. ${ }^{7}$

Kelly suggested testing calcium channel blockers next in patients with very rare forms of non-neuropathic LSDs for which there is no treatment. In any case, "studies in the standard animal knockout models of lysosomal storage disorders obviously cannot work here since the calcium channel blockers act to restore loss of enzymatic function," not to replace deficient enzyme.

Ernest Beutler, chairman of the department of molecular and experimental medicine at Scripps, also thinks that the next step should be studies in patients. In contrast to Kelly, however, Beutler thinks the first stop should be neuropathic LSDs and certain Gaucher's patients.

"Very little would be gained by performing further preclinical studies, and I believe the next step would be to perform clinical trials in patients with neuropathic disease and in patients homozygous for the N370S mutation who are not receiving enzyme replacement therapy," Beutler told SciBX. "There are many of the latter patients whose disease is sufficiently mild that one cannot justify giving them enzyme replacement therapy but who can be evaluated as research subjects receiving calcium-blocking agents."

However, Tony Futerman, professor in the department of biological chemistry at the Weizmann Institute of Science, cautioned that improving the overall folding capacity of the ER could potentially alter the activity of a broad range of enzymes. That could lead to unwanted side effects in a clinical setting, he told SciBX.

Kelly said quantitative proteomics studies are underway to determine to what extent the calcium channel blockers might influence the activity of multiple enzymes in the cell.

According to Frances Platt, reader in pharmacology at the University of Oxford, calcium channel blockers potentially could be combined with other LSD therapeutics. Indeed, "combination therapy with drugs that target different aspects of disease is the future of where therapy will go in lysosomal storage disorders, particularly those involving the brain," she said.

Platt and colleagues have shown that in mouse models of Sandhoff disease, Zavesca plus aspirin increased life expectancy by $73 \%$ compared with increases of $50 \%$ and $15 \%$ for Zavesca alone and aspirin alone $(p<0.00001){ }^{2}$ Some of the hallmarks of gangliosidosis LSDs such as Sandhoff and TaySachs are progressive microglial activation, macrophage recruitment and proinflammatory cytokine production in the brain. ${ }^{8}$ Anti-inflammatory drugs, such as aspirin, could therefore help slow disease progression.

David Lockhart, CSO of Amicus Therapeutics Inc., also expressed interest in the potential of combination therapies to treat LSDs. "It's exciting that another class of oral agents is being investigated for the treatment of LSDs," he told SciBX. "Because the calcium channel blockers work by a mechanism different from other LSD therapies, it is possible that they could be used as part of a combination with existing treatments or with other approaches under clinical development."

Lockhart noted the work is still very early.

"The new mechanism has been explored only in cells so far and at drug concentrations that may not be achievable in patients," he said. "And, as with any therapeutic agent being investigated for an entirely new indication and patient population, the risks and benefits of using calcium channel blockers in LSD patients will have to be carefully evaluated."

Amicus has a license from the Mount Sinai School of Medicine to patents and patent applications that cover the rescuing of mutant enzymes, enhancing enzyme activity to treat LSDs and specifically using isofagomine to treat Gaucher's disease.

The company is partnered with Shire plc to develop three chemical chaperones. Plicera isofagomine tartrate (AT2101), a chaperone that binds to glucocerebrosidase, is in a Phase II trial to treat type 1 Gaucher's. Amigal migalastat hydrochloride (AT1001), a chemical chaperone that binds $\alpha$-galactosidase, is in Phase II trials to treat Fabry's disease. Deoxynojirimycin (AT2220), a chemical chaperone that binds $\alpha$-glucosidase, is in a Phase I trial to treat Pompe's disease.

AT2101 and AT1001 have Orphan Drug designation from the FDA.

\section{REFERENCES}

1. Mu, T.-W. et al. PLoS Biol.; published online Feb. 5, 2008; doi:10.1371/ journal.pbio.0060026

Contact: Jeffery W. Kelly, The Skaggs Institute for Chemical Biology, The Scripps Research Institute, La Jolla, Calif.

e-mail: jkelly@scripps.edu

2. Jeyakumar, M. et al. Ann. Neurol. 56, 642-649 (2004)

3. Pastores, G. Recent Patents CNS Drug Discov. 1, 77-82 (2006)

4. Patterson, M. et al. Lancet Neurol. 6, 765-772 (2007)

5. Ginzburg, L. et al. Semin. Cell Dev. Biol. 15, 417-431 (2004)

6. Futerman, A. \& van Meer, G. Nat. Rev. Mol. Cell Biol. 5, 554-565 (2004)

7. Edelson, S. BioCentury 14(34), A13; July 31, 2006

8. Jeyakumar, M. et al. Brain 126, 974-987 (2003)

COMPANIES AND INSTITUTIONS MENTIONED

Actelion Ltd. (SWX:ATLN), Allschwil, Switzerland

Amicus Therapeutics Inc. (NASDAQ:FOLD), Cranbury, N.J. BioMarin Pharmaceutical Inc. (NASDAQ:BMRN; SWX:BMRN), Novato, Calif.

Genzyme Corp. (NASDAQ:GENZ), Cambridge, Mass.

Mount Sinai School of Medicine, New York, N.Y.

The Scripps Research Institute, La Jolla, Calif.

Shire plc (LSE:SHP; NASDAQ:SHPGY), Basingstoke, U.K.

University of Oxford, Oxford, U.K.

Weizmann Institute of Science, Rehovot, Israel 